\title{
Diversity in Mobile Communications for Blind Detection of Block-Coded Modulations
}

\author{
Gregori Vázquez, Francesc Rey, Meritxell Lamarca, Javier R. Fonollosa \\ Department of Signal Theory and Communications, Polytechnic University of Catalonia \\ UPC Campus Nord-Mòdul D5, c/ Jordi Girona 1-3, 08034 Barcelona (Spain) \\ E-mail: \{gregori,frey,xell, fono\}@gps.tsc.upc.es
}

\begin{abstract}
Spatial, temporal, and frequency diversity structures are analyzed to address the blind equalization problem in the presence of time-variant frequency selective channels. The aim of the paper is to present equalization schemes useful in front of fast changing channel responses. The best solution is a deterministic blind criterion that allows direct channel equalization and symbol detection.

The main contribution of this paper is to present deterministic blind equalization schemes in CDMA systems (frequency diversity) to reduce the impact of the time-variant frequency selective channel.
\end{abstract}

\section{INTRODUCTION}

The issue of developing equalization techniques in mobile communications has received considerable attention recently. The time-variant nature of the channel behavior suggests the definition of deterministic algorithms instead of the use of conventional stochastic ones. On the other hand, spread spectrum technology is in special interest in the presence of frequency selective fading channels. The pseudo-noise (PN) sequence, or spreading code, allows the receiver to reduce the channel attenuation effect in certain frequencies.

It is known that if some diversity structures are introduced in transmission and/or reception, the channel effects over the information are reduced, increasing the communication system robustness. This paper deals with frequency diversity, temporal diversity and spatial diversity. Frequency diversity, also known as spread spectrum, is introduced by the PN sequence when spreading the message information. Temporal diversity can be achieved when the channel output is oversampled in the time domain, or in OFDM schemes. Finally to obtain spatial diversity multiple antennas in transmission and/or reception

\footnotetext{
* This work has been partially supported by the Spanish government (TIC96-0500-C10-01, TIC98-0412, TIC98-0703) and the Catalan govemment (CIRIT 1998SGR-00081).
}

must be used. In [1] and [2] equalizers based on a deterministic criterion have been developed to detect the transmitted information using spatial and/or temporal diversity at the receiver.

This paper proposes a blind equalization algorithm of CDMA systems in multipath frequency selective channels. Our contribution combines spread spectrum technologies (frequency diversity) with deterministic blind equalizers based on temporal diversity. The next section presents spread spectrum diversity and defines the equivalent channel to equalize after the despreading when the signal is oversampled. Section 3 summarizes two deterministic equalization criteria that exploit spatial and/or temporal diversity to detect the transmitted information. In section 4 some simulation results that evaluate the performance of the proposed schemes are presented. Finally in section 5 some conclusions of the present work are drawn.

\section{SPREAD SPECTRUM DIVERSITY}

Spread Spectrum diversity, also called frequency diversity or multipath diversity, is introduced by the pseudo-noise (PN) sequence when spreads the data stream information in a wide band spectrum. This kind of diversity is in special interest in the presence of frequency-selective fading channels, allowing the receiver to recover the transmitted information although the channel might introduce attenuation in certain frequencies.

Figure 1 illustrates the scheme of a DS-CDMA system. The data sequence $x(t)$ is spread by the user signature waveform $b(t)$ and transmitted through the channel. The processing gain is given by $G_{p}=T / T_{c}$, where $T$ is the symbol duration, and $T_{c}$ is the chip period.

In a simplified DS-CDMA scheme, the received signal is the transmitted signal degraded by an $A W G N$ term. After the received signal de-spreading and integrating it over a symbol interval $T$, an estimation of the transmitted symbol is obtained. But in a real wireless communications mobile scenario we have to consider the problem of digital signaling over a frequency- 
selective channel $h(t)$, in this case it is necessary to design receiver structures capable to combat multipath. Let as consider the existence of intersymbol interference (ISI) due to channel response duration $T_{m}$ higher than the symbol interval $T$.

The proposed scheme (figure 1) despreads the received signal and uses an equalizer to suppress the ISI introduced by the multipath channel. Notice that after the despreading, if the signal is oversampled at $B$ samples per symbol, with $B \leq G_{p}$, and an equalizer that exploits this kind of temporal diversity can be used.

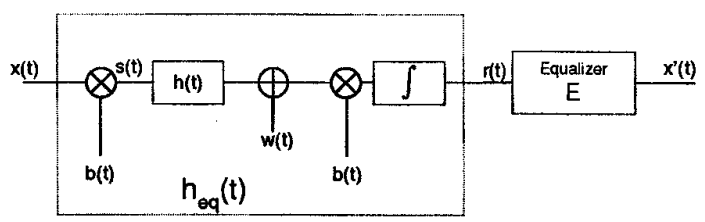

Figure 1. General scheme

Finally it is important to remark that, as illustrated in figure 1 , the equalizer will not equalize directly the multipath channel $h(t)$ but an equivalent channel including spreading, frequency-selective channel distortion and despreading processes. This equivalent channel $h_{e q}(t)$, neglecting the AWGN term, can be expressed as:

$$
h_{e q}(t)=(b(t) * h(t)) \cdot b(t)
$$

\section{SPATIAL AND TEMPORAL DIVERSITY}

Blind spatial and/or temporal diversity based equalizers in digital receivers with a single-input multiple-output formulation (SIMO) have been presented in works [1] and [2]. Let as consider the discrete-time signal model for temporal and spatial diversity receivers as depicted in figures $1 \mathrm{a}$ and $\mathbf{1 b}$ respectively. In the time diversity receiver, the information signal $T[k]$ is transmitted through a mobile channel response $C[k]$, which distorts the signal and degrades it by an AWGN term $W[k]$. The received signal is oversampled at $B$ samples per symbol, and introduced in $B$ different branches. In a similar way the spatial diversity receiver transmits the information signal $T[k]$ through $B$ diversity branches, it is distorted by $B$ different channel responses $C[k]$ and finally degraded by $B$ noise terms $W^{k}[k]$.

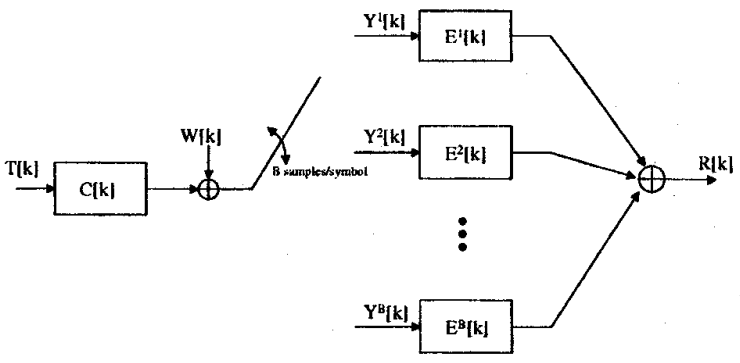

Figure 2a. Block diagram of the temporal diversity system.

The equalization process for spatial and temporal diversity schemes is similar, and both can be combined into the same receiver system. Their associated equations can be written in the $\mathrm{z}$-transform domain as:

$$
Y^{i}(z)=T(z) C^{i}(z)+W^{i}(z) \quad i=1, \ldots, B
$$

As shown in [3], the equalization process can be designed following a blind criterion. The multiple temporal or spatial diversity branches are combined by means of FIR filters $E[k]$ to generate an output $R[k]$ :

$$
\begin{aligned}
R(z) & =\sum_{i=1}^{B} Y^{i}(z) E^{i}(z)= \\
& =T(z) \sum_{i=1}^{B} C^{i}(z) E^{i}(z)+\sum_{i=1}^{B} W^{i}(z) E^{i}(z)
\end{aligned}
$$

Under noise-free conditions, the perfect equalization criterion requires $R(z)=T(z)$, and therefore :

$$
\sum_{i=1}^{B} C^{f}(z) E^{\prime}(z)=1
$$

The Bezout equation [4] guarantees that the previous equation has solution if and only if the $B$ channel responses have no common zeros, or in other words

$$
T(z)=g \cdot c \cdot d \cdot\left\{Y^{i}(z)\right\}
$$

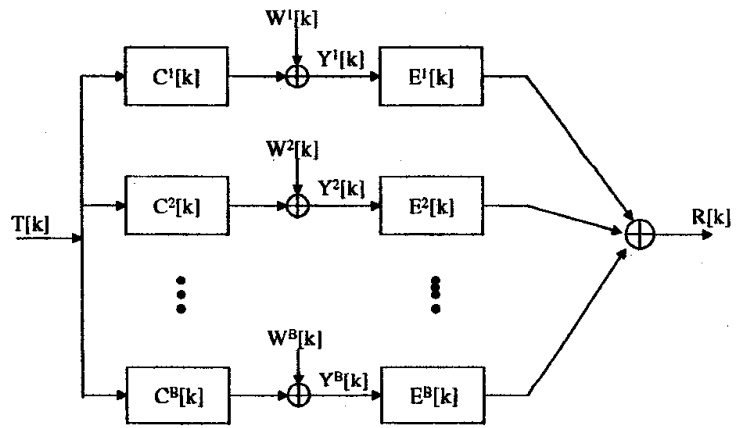

Figure $2 \mathrm{~b}$. Block diagram of the spatial diversity system. 
Two different design criteria have been proposed in [1] and [2] to obtain the equalizer coefficients in (3). Although the cost function in both cases is similar, the philosophy that guaranties the perfect equalization criterion (equation (4)) is completely different. The first algorithm observes the complete convolution of the transmitted data to obtain the channel information, while the second criterion is based on the redundancy of linear-block codes to characterize the channel response. Both methods are briefly summarized here in order to approach the problem:

As shown in [3], equation (3) can be written using a matrix notation as:

$$
\mathbf{r}=\mathbf{Y e}
$$

where $\mathbf{r}$ is the equalizer output vector, $\mathbf{Y}$ is a Sylvester matrix with the received data and $\mathrm{e}$ is the equalizer weight vector. The perfect equalization noise-free case can be written in a matrix notation as:

$$
\mathbf{r}=\alpha\left[\begin{array}{l}
\mathbf{t} \\
0
\end{array}\right]=\left[\begin{array}{l}
\mathbf{Y}_{\mathbf{t}} \\
\mathbf{Y}_{0}
\end{array}\right] \mathbf{e}
$$

where the received data matrix $\mathbf{Y}$ has been split in two parts

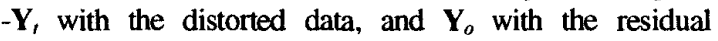
sequence due to the convolution with the channel response ('tails ), $\mathbf{t}$ is the transmitted data vector and $\alpha$ is an arbitrary multiplicative constant.

In [1] a blind scheme was suggested such that the signal-toISI-plus-noise-ratio (SINR) at the equalizer output is maximized, that is:

$$
\operatorname{SINR}=\frac{\mathbf{e}^{H} \mathbf{Y}_{\mathbf{t}}{ }^{H} \mathbf{Y}_{\mathbf{t}} \mathbf{e}}{\mathbf{e}^{H} \mathbf{Y}_{0}{ }^{H} \mathbf{Y}_{0} \mathbf{e}}
$$

In noisy environments many stability problems with the $Y_{o}$ matrix may appear. In order improve the algorithm robustness [2] proposes a different cost function obtaining a new design criteria, which avoids the use of the residual $Y_{o}$ matrix. This new cost function uses the information contained in the redundancy of linear-block in order to improve the algorithm robustness. Equation (9) depicts the construction of linear block codes using matrix notation. It describes how to encode a $k$-symbols data information vector $t$ using the code generator $n x k$ matrix $\mathbf{G}_{\mathbf{c}}$ to obtain the $n$-symbols code word $\mathbf{t}^{\prime}$ :

$$
t^{\prime}=G_{c} t
$$

To ensure that the encoding process maintains constant the symbol energy of all the transmitted symbols we are interested on those transform such that:

$$
\left|g_{1}\right|^{2}=\left|g_{2}\right|^{2}=\ldots=\left|g_{n}\right|^{2}=1
$$

where $\left|\mathbf{g}_{\mathbf{i}}\right|$ is the Euclidean norm of the $i$-th row vector of $\mathbf{G}_{\mathbf{c}}$ (a more accurate study on the generator matrix as well as the structure for systematic linear block codes is detailed in [5]).

According to (9), the encoding matrix $\mathbf{G}_{\mathbf{c}}$ is considered as a $n x k(n>k)$ full rank linear transform in the data encoding process[6],[7]; and so, vector 't' is contained in the signal subspace $S$ spanned by the $k$ columns of matrix $\mathbf{G}_{\mathbf{c}}$.

Let as consider the orthogonal subspace $S^{\perp}$ spanned by an $r=n-k$ dimensional orthogonal basis and its associated generation matrix $\mathbf{G}_{\mathfrak{c}}{ }^{1}$ (check matrix). By definition it accomplishes:

$$
\mathbf{G}_{\mathrm{c}}^{\perp} \mathbf{G}_{\mathbf{c}} \mathbf{t}=\mathbf{G}_{\mathbf{c}}^{\perp} \mathbf{t}^{\prime}=\mathbf{0}
$$

The aim of the check matrix will be to detect changes between the transmitted code and the received information. The outputs of the marginal channels $\mathbf{C}^{i}(i=1,2, \ldots, B)$ to the transmitted data $\mathbf{t}^{\prime}$ and the channel noise contributions $w^{i}$ will force the received data to be contained in the $S \oplus S^{\perp}$. Basically, the projection of the received data in the orthogonal subspace $S^{\perp}$ is used by the equalizer to characterize the channel response and noise distribution.

Thus, the two new equalizer design equations becomes:

$$
\begin{aligned}
& \mathbf{t}^{\prime}=Y_{t} \mathbf{e} \\
& C_{c} t^{\prime}=C_{c} Y_{t} e=0
\end{aligned}
$$

According to (12), the new SNIR estimate is formulated as:

$$
\operatorname{SINR}{ }^{\prime}=\frac{\mathrm{e}^{H} \mathbf{Y}_{\mathrm{t}}{ }^{H} \mathbf{Y}_{\mathrm{t}} \mathbf{e}}{\mathbf{e}^{H} \mathbf{Y}_{\mathrm{t}}{ }^{H} \mathbf{C}_{\mathbf{c}}{ }^{H} \mathbf{C}_{\mathbf{c}} \mathbf{Y}_{\mathrm{t}} \mathbf{e}}
$$

This new cost function to be optimized is more robust in presence of AWGN because the residual $\mathbf{Y}_{o}$ matrix is not needed. According to equation (13), the equalizer that maximizes the new signal to noise plus $I S I$ ratio corresponds to the generalized eigenvector associated with the maximum generalized eigenvalue:

$$
\mathbf{Y}_{\mathbf{t}}{ }^{H} \mathbf{Y}_{\mathbf{t}} \mathbf{e}=\lambda_{\max } \mathbf{Y}_{\mathbf{t}}{ }^{H} \mathbf{C}_{\mathbf{c}}{ }^{H} \mathbf{C}_{\mathrm{c}} \mathbf{Y}_{\mathbf{t}} \mathbf{e}
$$

As usual, the equalization performance can be optimized if a delay is allowed in $R(z)$ in equation (3), and the best equalizer is selected as that one which yields the greatest $\lambda_{\max }$ (maximum SINR in equation (8) and maximum $S I N R^{\prime}$ in equation (13)). 


\section{SIMULATION RESULTS}

In this section we present the performance of spread spectrum diversity in time-variant frequency selective channels using equalizers (8) and (13). The simulations display the percentage of realizations, over 1000 realizations, for which the equalizer output EbNo was higher than the value indicated in the $\mathrm{x}$-axis. In all cases 8 chip PN sequences (based on 7 length Gold codes, plus an additional chip $( \pm 1)$ computed to guarantee the zero mean) with rectangular pulse shaping were considered. The channel EbNo was $15 \mathrm{~dB}$, the order of the diversity (temporal diversity) $\mathrm{B}=8$, and the FIR filters in each branch of the equalizer had four coefficients. When using redundancy 118 QPSK data symbols plus 10 redundancy symbols generated with a sub-Hadamard matrix were transmitted, while when no redundancy was used, the transmitted information length was 128 QPSK symbols.

The frequency selective multipath channel response is presented in figure 3 and table 1 . This model has been extracted from the ETSI document [8], and corresponds with a vehicular scenario. The multipath spread of the channel $T_{m}=2684 \mathrm{~ns}$ is higher than the symbol period $T=976 \mathrm{~ns}$, and so ISI must be considered.

In figure 4 the performance of both equalizers is compared with the RAKE correlator [9]. Although the RAKE receiver is the optimum receiver for processing a spread spectrum signal, the proposed scheme outperforms in some cases this correlator. It can be justified because the RAKE receiver assumes ideal autocorrelation properties of the PN spreading sequence, and neglects the ISI considering $T \gg T_{m}$. Another advantage of the proposed scheme is that it is a blind algorithm, whereas the RAKE receiver needs to estimate the channel coefficients (in our case we have considered them as perfectly known).

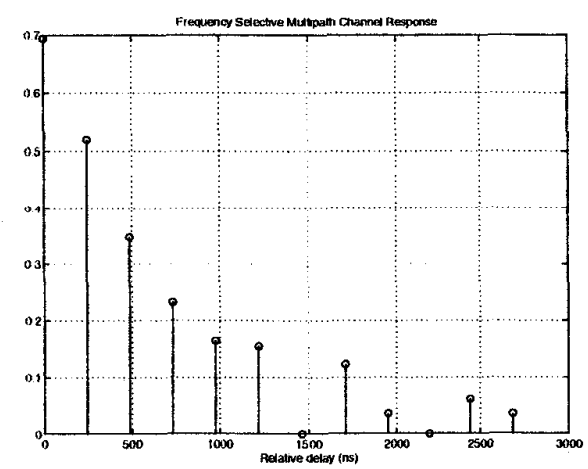

Figure 3. Channel Response (modulus).

\begin{tabular}{|c|c|c|}
\hline Tap & Modulus & $\begin{array}{c}\text { Phase } \\
\text { (Unif. [0 2 } \pi \text { ) }\end{array}$ \\
\hline 1 & 0.6938 & 1.7747 \\
\hline 2 & 0.5203 & 0.4274 \\
\hline 3 & 0.3477 & 5.2840 \\
\hline 4 & 0.2324 & 5.3605 \\
\hline 5 & 0.1645 & 2.8586 \\
\hline 6 & 0.1553 & 4.3292 \\
\hline 7 & - & - \\
\hline 8 & 0.1234 & 0.4776 \\
\hline 9 & 0.0368 & 0.9652 \\
\hline 10 & - & - \\
\hline 11 & 0.0618 & 0.6533 \\
\hline 12 & 0.0368 & 3.4644 \\
\hline & Table 1. Channel Response. \\
\hline
\end{tabular}

Notice that as shown in (1) the equivalent channel to equalize $h_{e q}(t)$ depends on the PN sequence, thus different sequences will provide different performances. All possible $\mathrm{PN}$ sequences have been simulated for (8) and (13). Figure 5 presents the performance for the best and the worst code for (8) (without the use of linear block codes). Figure 6 presents the upper and lower bounds in the performance for (13) (with linear block codes). The performance improvement of (13) over (8) was already presented in [2] and [5], where the algorithm is applied in TDMA structures.

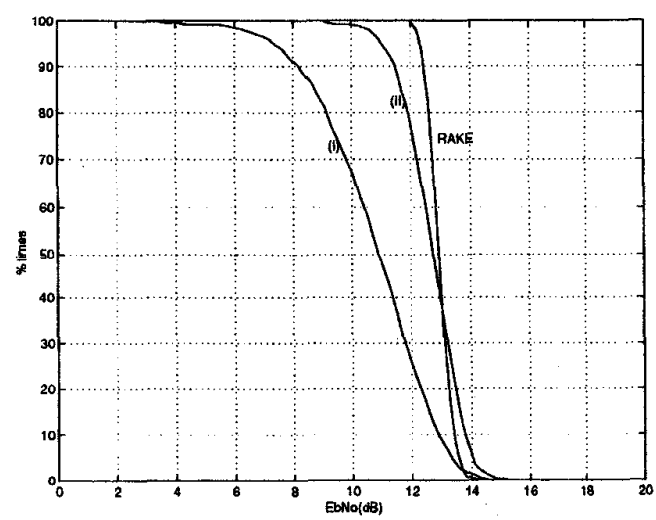

Figure 4. Algorithm performance vs RAKE receiver. Blind equalizer without (I) and with (II) the use of redundancy. 


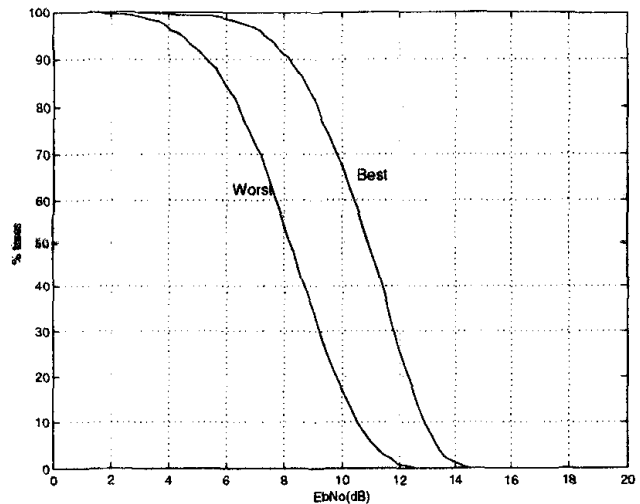

Figure 5. Upper and lower performance bounds for different PN sequences. Blind equalizer without the use of redundancy. Cost function (8).

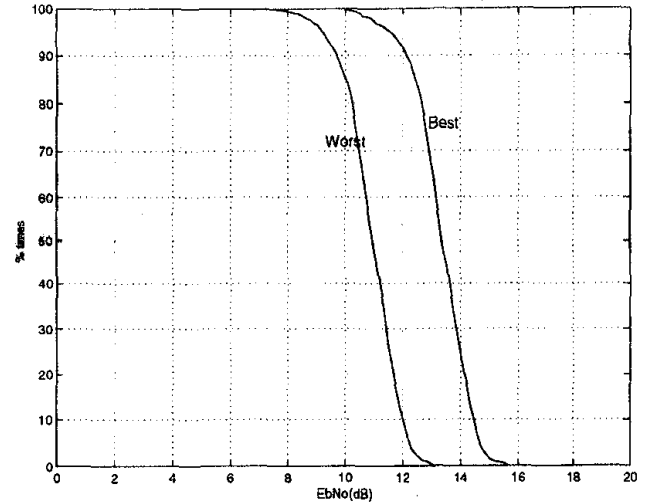

Figure 6. Upper and lower performance bounds for different PN sequences. Blind equalizer with the use of redundancy. Cost function (13).

\section{REFERENCES}

Finally we want to remark that in deterministic algorithms the performance of the equalizer are not only dependent on the PN sequence but also on the information data realization, the channel realization and the noise realization in a burst. Thus some performance differences can appear under different channel responses.

\section{CONCLUSIONS}

A blind equalization scheme for CDMA systems in multipath frequency selective channels has been introduced. This paper is the natural extension of previous works done in [1] and [2], where equalizers based on a deterministic criterion were developed to directly identify the transmitted signal, by means of spatial and/or temporal diversity. The new scheme combines those equalization techniques with the spread spectrum diversity introduced by CDMA systems.

The proposed equalization scheme over DS-CDMA systems, using the temporal diversity equalization techniques presented in [1] and [2], have been evaluated and compared with the RAKE receiver. The result is an algorithm that, although it is very sensitive to the channel response and the spreading signature, can be in special interest when the channel estimation is not possible or it is difficult due to its time-variant nature. Under those conditions a blind equalization criterion can performs better than a RAKE receiver does.
[1] M. Lamarca, G. Vázquez, 'Diversity techniques for blind channel equalization in mobile communications', Proceedings. of PIMRC'97, pp. 1079-1083, Helsinki (Finland), Sept. 1997.

[2] F. Rey, M. Lamarca, G. Vázquez, 'Blind Equalization Based on Spatial and Temporal Diversity in Block Coded Modulations', Proc. of PIMRC'98, Boston (USA), Sept. 1998.

[3] M. Lamarca, G. Vázquez, 'Multichannel receivers for OFDM and TDMA in mobile communications'. Proc. of ICASSP '97, Munich (Germany), April. 1997.

[4] Y.lil, Z.Ding, 'Blind channel identification based on second order cyclostacionary statistics', Proceedings. of ICASSP'93 Minneapolis (USA)

[5] F. Rey, M. Lamarca, G. Vázquez, 'Redundancy in Block Coded Modulations for Channel Equalization Based on Spatial and Temporal Diversity', Proceedings. of ICASSP'99, Phoenix (USA), March 1999.

[6] M. Lamarca, G. Vázquez, "Transform modulations in mobile communications', Proc. of PIMRC'97, pp. 462-466, Helsinki (Finland), Sept. 1997.

[7] M. Lamarca, G. Vázquez, 'Channel estimation for transform modulations in mobile communications', Proc. of EUSIPCO'96, Trieste (Italy), Sept. 1996.

[8] 'Submission of Proposed Radio Transmission Technologies: the ETSI UMTS Terrestrial Radio Access (UTRA) ITU-R RTT Candidate Submission', ETSI SMG2. Date of submission: 29/1/1998. (Available at the ITU WWW http://www.itu.ch/imt/).

[9] J.G. Proakis, 'Digital Communications, 3rt Ed.', McGraw Hill 1995. 